\title{
Analysis of an Underground Horizontal Electrically Small Antenna
}

\author{
Shuwei Dong, Aiguo Yao, and Fanhe Meng \\ Faculty of Engineering, China University of Geosciences, Wuhan 430074, China \\ Correspondence should be addressed to Aiguo Yao; a.yao@cug.edu.cn
}

Received 24 January 2015; Revised 24 March 2015; Accepted 3 April 2015

Academic Editor: Xianming Qing

Copyright ( 2015 Shuwei Dong et al. This is an open access article distributed under the Creative Commons Attribution License, which permits unrestricted use, distribution, and reproduction in any medium, provided the original work is properly cited.

\begin{abstract}
This work is about a horizontal electrically small wire antenna located underground, which transmits electromagnetic signals to the ground. To solve this problem, the expressions of the magnetic vector potential and the electric field are derived. Further, a quasi-static situation is considered in the condition of extremely low frequency (ELF) or super low frequency (SLF) to make an approximation on Sommerfeld integral for easy calculation. The method of moments (MOM) is used to solve the current distribution along the antenna surface at different frequencies, which lays a good foundation for obtaining the electric field of the antenna. Then the three components of the electric field along the radial distance at different polar angles on the ground are investigated, as well as the voltage received on the ground. Furthermore, some influence factors of the antenna are analyzed in order to know the operating characteristics of the antenna better. The results indicate that the antenna length and relative permittivity have a positive correlation with the radial component of the electric field magnitude, while the buried depth, frequency, and conductivity have a negative correlation with that. The influence of these factors on electric field is obvious except the relative permittivity.
\end{abstract}

\section{Introduction}

Electromagnetic underground communication plays an important role in the mine production. Through the stratum, the communication system can propagate signals from underground to ground, the kernel technology of which is the use of underground antenna. Unlike conventional communication, the underground communication faces a series of difficulties because the transmission medium is the lossy formation, which will cause attenuation of electromagnetic waves. The issue of electromagnetic propagation through different media has been studied first by Sommerfeld [1] in 1909. Then Sommerfeld [2] established a vertical dipole model placed at the surface of ground and used the evaluation of FourierBessel integrals to solve it. Moore and Blair [3] gave the field expressions of a dipole near a conducting half space in 1961. Bannister [4-6] did a series of studies about quasi-static field of antennas at or above ground using complex image theory and made a conclusion of his achievements in 1979. Chang and Wait [7] did the study of a horizontal wire antenna which is located above or buried in the earth with extremely low frequency and derived explicit expressions for the propagation constant in 1974. King [8] did the study about a dipole over an imperfectly conducting half space as early as 1990. In 1997, King et al. [9] studied a very low frequency antenna in the sea and gave the results of electromagnetic fields. Tai and Collin [10] researched the radiation of a dipole immersed in a dissipative medium in 2000. In 2004, Thottappillil et al. [11] gave the formulas of a semi-infinite antenna above a conducting plane. Vong et al. [12] modeled an electromagnetic telemetry system and gave a portion of results in 2005. Xu and Huang [13] used an efficient computation to solve the problem of an electric dipole above a lossy half space in 2007. Khalatpour et al. [14] used numerical methods to study a wire antenna above a lossy half space in 2010. Parise [15] did a research on quasistatic theory and obtained the harmonic electromagnetic field components excited by a vertical electric dipole lying on the surface of a flat and homogeneous lossy half space in 2013. In addition, there are many scholars who have done researches on related problems. However, the detailed study 
of a horizontal electrically small antenna in a lossy medium has been not common in previous researches. A horizontal electrically small antenna with ELF or SLF is the best choice for underground electromagnetic communication generator, which can be used effectively in mine communication. This kind of antenna can play a good role in other areas such as energy development, trenchless technology, measurement while drilling, and lateral wells butt.

In this paper, an accurate model about a horizontal electrically small antenna located underground with ELF or SLF has been proposed. Analytical solutions of the field excited by underground antenna have been obtained to solve this problem. Sommerfeld integral in the expressions can cause some troubles on calculation, so quasi-static approximation has been used to overcome the difficulties in this low frequency problem. The main work is to get the induction field of the electrically small antenna; for this purpose, numerical methods including the MOM have been used. Numerical results such as the current distribution and the electric field have been obtained in order to describe the antenna character. As a further study, the effect of inside and outside parameters on induction field has been considered and the variation curves of the current and the electric field with the influence factors have been got for better understanding of the antenna.

\section{Mathematical Procedure}

Assume that the earth is homogeneous medium; the magnetic vector potential A satisfies (1) obtained from the Maxwell's equations:

$$
\nabla^{2} \mathbf{A}+k^{2} \mathbf{A}=-\mu \mathbf{J}
$$

where $\mathbf{J}$ is the current density of an impressed source, $k$ is the wave number given by $k^{2}=-i \omega \mu(\sigma+i \omega \varepsilon), \varepsilon, \mu$, and $\sigma$ are, respectively, the permittivity, permeability, and conductivity of the medium, and $\omega$ is the angular frequency. What should be noted is that the time dependence $e^{i \omega t}$ is assumed and suppressed in this equation and the following analysis.

As shown in Figure 1, the air occupies the upper half space referred to as medium 0 whereas the ground occupies the lower one referred to as medium 1 , and the coordinate origin is at the interface. The horizontal electric dipole is oriented in the $x$ direction and located at the coordinates $(0,0,-h)$, while the coordinates of its image are $(0,0, h)$, where $h>0$. Considering that the dipole is represented by the current density $\mathbf{J}=I l \delta\left(\mathbf{r}-\mathbf{r}^{\prime}\right) \mathbf{e}_{x}$, the magnetic vector potentials in two media satisfy the following two equations, respectively:

$$
\begin{aligned}
& \nabla^{2} \mathbf{A}_{0}+k_{0}^{2} \mathbf{A}_{0}=0 \\
& \nabla^{2} \mathbf{A}_{1}+k_{1}^{2} \mathbf{A}_{1}=-\mu I l \delta\left(\mathbf{r}-\mathbf{r}^{\prime}\right) \mathbf{e}_{x} .
\end{aligned}
$$

From the boundary conditions, there are two components of the magnetic vector potential oriented in $x$ and $z$ direction, respectively. The field generated by the horizontal dipole is the superposition of the primary field and the reflected field of the source, which can be derived by the image theory. According

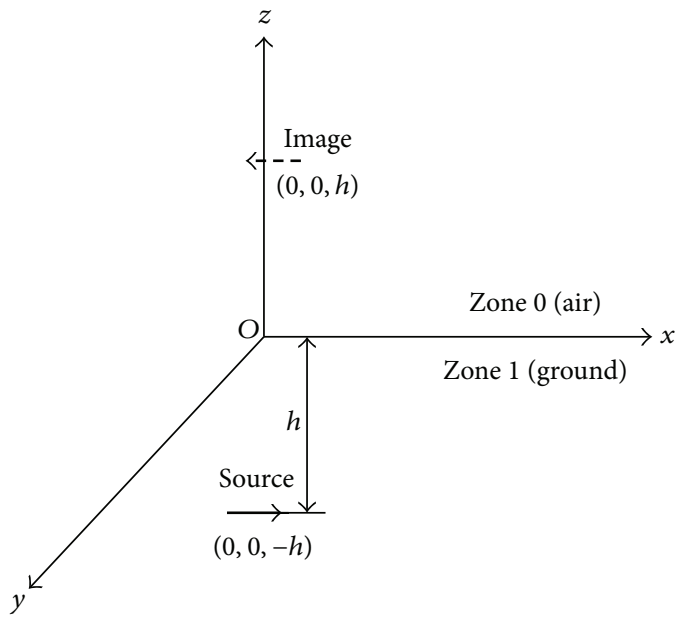

FIGURE 1: Schematic of the horizontal electric dipole.

to what we have known of the magnetic vector potentials in free-space, the $x$ component of the vector potentials in two media satisfies (4) according to the image theory [16]:

$$
\begin{aligned}
& A_{0 x}=\frac{\mu I l}{4 \pi} \int_{0}^{\infty} P \frac{k_{\rho}}{\xi_{0}} e^{-\xi_{0}|z+h|} J_{0}\left(k_{\rho} \rho\right) d k_{\rho}, \\
& A_{1 x} \\
& =\frac{\mu I l}{4 \pi} \int_{0}^{\infty}\left(\frac{k_{\rho}}{\xi_{1}} e^{-\xi_{1}|z+h|}+Q \frac{k_{\rho}}{\xi_{1}} e^{-\xi_{1}|z-h|}\right) J_{0}\left(k_{\rho} \rho\right) d k_{\rho},
\end{aligned}
$$

where $P$ and $Q$ are undetermined coefficients determined by the boundary conditions and $J_{0}$ is the zero-order Bessel function of the first kind. Here some variables are given by $\rho^{2}=x^{2}+y^{2}, k_{\rho}^{2}=k_{x}{ }^{2}+k_{y}{ }^{2}, \xi_{0}{ }^{2}=k_{\rho}{ }^{2}-k_{0}{ }^{2}$, and $\xi_{1}{ }^{2}=k_{\rho}{ }^{2}-k_{1}{ }^{2}$, where $k_{0}$ and $k_{1}$ are, respectively, the wave numbers of the air and the ground.

The boundary conditions are given by (5), from which the relationship equations between $P$ and $Q$ can be derived:

$$
\begin{gathered}
\lim _{z \rightarrow 0+} A_{0 x}=\lim _{z \rightarrow 0-} A_{1 x}, \\
\lim _{z \rightarrow 0+} \frac{\partial A_{0 x}}{\partial z}=\lim _{z \rightarrow 0-} \frac{\partial A_{1 x}}{\partial z} .
\end{gathered}
$$

Further, the coefficients $P$ and $Q$ are obtained as the following expressions:

$$
\begin{aligned}
& P=\frac{2 \xi_{0}}{\xi_{1}+\xi_{0}} e^{\left(\xi_{0}-\xi_{1}\right) h}, \\
& Q=\frac{2 \xi_{1}}{\xi_{1}+\xi_{0}}-1 .
\end{aligned}
$$


So far, the $x$ component of the vector potentials in two media can be expressed as the following expressions:

$$
\begin{aligned}
A_{0 x} & =\frac{\mu I l}{4 \pi} I_{0 x}, \\
A_{1 x} & =\frac{\mu I l}{4 \pi}\left(\frac{e^{-i k_{1} R}}{R}-\frac{e^{-i k_{1} R_{i}}}{R_{i}}+I_{1 x}\right), \\
I_{0 x} & =\int_{0}^{\infty} \frac{2 k_{\rho}}{\xi_{1}+\xi_{0}} e^{-\xi_{0} z-\xi_{1} h} J_{0}\left(k_{\rho} \rho\right) d k_{\rho}, \\
I_{1 x} & =\int_{0}^{\infty} \frac{2 k_{\rho}}{\xi_{1}+\xi_{0}} e^{\xi_{1}(z-h)} J_{0}\left(k_{\rho} \rho\right) d k_{\rho},
\end{aligned}
$$

where $R=\sqrt{\rho^{2}+(z+h)^{2}}$ and $R_{i}=\sqrt{\rho^{2}+(z-h)^{2}}$.

The following work is to solve the $z$ component of the vector potentials. Since it is independent of the source, the wave equations (11) can be got:

$$
\begin{aligned}
& \nabla^{2} A_{0 z}+k_{0}{ }^{2} A_{0 z}=0, \\
& \nabla^{2} A_{1 z}+k_{1}{ }^{2} A_{1 z}=0 .
\end{aligned}
$$

According to the knowledge of partial differential equations, the general solutions of (11) in a cylindrical coordinate system $(\rho, \theta, z)$ are as follows:

$$
\begin{aligned}
& A_{0 z} \\
& \quad=\frac{\mu I l}{4 \pi} \sum_{n=-\infty}^{\infty} e^{i n \theta} \int_{0}^{\infty}\left(a_{0 n} e^{-\xi_{0} z}+b_{0 n} e^{\xi_{0} z}\right) J_{n}\left(k_{\rho} \rho\right) d k_{\rho}, \\
& A_{1 z} \\
& \quad=\frac{\mu I l}{4 \pi} \sum_{n=-\infty}^{\infty} e^{i n \theta} \int_{0}^{\infty}\left(a_{1 n} e^{-\xi_{1} z}+b_{1 n} e^{\xi_{1} z}\right) J_{n}\left(k_{\rho} \rho\right) d k_{\rho} .
\end{aligned}
$$

Because of $\lim _{z \rightarrow \infty} e^{\xi_{0} z}=0$ and $\lim _{z \rightarrow-\infty} e^{-\xi_{1} z}=0$, in order to ensure that $A_{0 z}$ and $A_{1 z}$ are finite, the coefficients $b_{0 n}$ and $a_{1 n}$ must be 0 . Meanwhile, the vector potential satisfies $A_{0 z}(\rho, \theta, z)=A_{0 z}(\rho,-\theta, z)$ and $A_{1 z}(\rho, \theta, z)=A_{1 z}(\rho,-\theta, z)$, so $A_{0 z}$ and $A_{1 z}$ are expressed by

$$
\begin{aligned}
& A_{0 z}=\frac{\mu I l}{4 \pi} \sum_{n=-\infty}^{\infty} \cos \theta \int_{0}^{\infty} a_{n} e^{-\xi_{0} z} J_{n}\left(k_{\rho} \rho\right) d k_{\rho}, \\
& A_{1 z}=\frac{\mu I l}{4 \pi} \sum_{n=-\infty}^{\infty} \cos \theta \int_{0}^{\infty} b_{n} e^{\xi_{1} z} J_{n}\left(k_{\rho} \rho\right) d k_{\rho} .
\end{aligned}
$$
follows:

The boundary conditions related to $A_{0 z}$ and $A_{1 z}$ are as

$$
\begin{aligned}
& \lim _{z \rightarrow 0+} A_{0 z}=\lim _{z \rightarrow 0-} A_{1 z} \\
& \lim _{z \rightarrow 0+} \frac{1}{k_{0}^{2}}\left(\frac{\partial A_{0 x}}{\partial x}+\frac{\partial A_{0 z}}{\partial z}\right) \\
& =\lim _{z \rightarrow 0-} \frac{1}{k_{1}^{2}}\left(\frac{\partial A_{1 x}}{\partial x}+\frac{\partial A_{1 z}}{\partial z}\right) .
\end{aligned}
$$

By using the boundary conditions, the $z$ component of the vector potentials in two media is got, respectively:

$$
\begin{aligned}
& A_{1 z}=\frac{\mu I l \cos \theta}{4 \pi} I_{1 z}, \\
& A_{0 z}=\frac{\mu I l \cos \theta}{4 \pi} I_{0 z}, \\
& I_{0 z} \\
& =\int_{0}^{\infty} \frac{2\left(k_{0}{ }^{2}-k_{1}{ }^{2}\right) k_{\rho}{ }^{2}}{\left(\xi_{1}+\xi_{0}\right)\left(k_{0}{ }^{2} \xi_{1}+k_{1}{ }^{2} \xi_{0}\right)} e^{-\xi_{1} h-\xi_{0} z} J_{1}\left(k_{\rho} \rho\right) d k_{\rho}, \\
& I_{1 z} \\
& =\int_{0}^{\infty} \frac{2\left(k_{0}{ }^{2}-k_{1}{ }^{2}\right) k_{\rho}{ }^{2}}{\left(\xi_{1}+\xi_{0}\right)\left(k_{0}{ }^{2} \xi_{1}+k_{1}{ }^{2} \xi_{0}\right)} e^{\xi_{1}(z-h)} J_{1}\left(k_{\rho} \rho\right) d k_{\rho} .
\end{aligned}
$$

The magnetic vector potential underground is obtained so far, and it is

$$
\mathbf{A}_{1}=\frac{\mu I l}{4 \pi}\left[\left(\frac{e^{-i k_{1} R}}{R}-\frac{e^{-i k_{1} R_{i}}}{R_{i}}+I_{1 x}\right) \mathbf{e}_{x}+\cos \theta I_{1 z} \mathbf{e}_{z}\right] .
$$

In the following analysis, what we are concerned about is the vector potential underground presented as $\mathbf{A}_{1}$. For simplicity, the subscript 1 will be omitted. From Maxwell's equations, the relationship expression between the electric field $\mathbf{E}$ and the vector potential $\mathbf{A}$ is shown as

$$
\mathbf{E}=-i \omega \mathbf{A}+\frac{1}{\mu \sigma+i \omega \mu \varepsilon} \nabla \nabla \cdot \mathbf{A} .
$$

To solve the electric field E, $\nabla \cdot \mathbf{A}$ should be solved first. Since $A_{\rho}=\cos \theta A_{x}$ and $A_{\theta}=-\sin \theta A_{x}$, the expression of $\nabla \cdot \mathbf{A}$ can be derived in cylindrical coordinate system $(\rho, \theta, z)$ :

$$
\nabla \cdot \mathbf{A}=\frac{1}{\rho} \frac{\partial\left(\rho A_{\rho}\right)}{\partial \rho}+\frac{1}{\rho} \frac{\partial A_{\theta}}{\partial \theta}+\frac{\partial A_{z}}{\partial z}=\frac{\partial A_{\rho}}{\partial \rho}+\frac{\partial A_{z}}{\partial z} .
$$

By using the recurrence formula $(\partial / \partial \rho) J_{0}\left(k_{\rho} \rho\right)=$ $-k_{\rho} J_{1}\left(k_{\rho} \rho\right)$, the following result is acquired:

$$
\begin{aligned}
& \frac{\partial A_{z}}{\partial z}=-\frac{\mu I l \cos \theta}{4 \pi} \frac{\partial}{\partial \rho} I_{1 z^{\prime}} \\
& I_{1 z^{\prime}} \\
& \quad=\int_{0}^{\infty} \frac{2\left(k_{0}{ }^{2}-k_{1}{ }^{2}\right) k_{\rho} \xi_{1}}{\left(\xi_{1}+\xi_{0}\right)\left(k_{0}{ }^{2} \xi_{1}+k_{1}{ }^{2} \xi_{0}\right)} e^{\xi_{1}(z-h)} J_{0}\left(k_{\rho} \rho\right) d k_{\rho} .
\end{aligned}
$$

Then

$$
\begin{aligned}
\nabla \cdot \mathbf{A} & =\frac{\mu I l}{4 \pi} \cos \theta \frac{\partial}{\partial \rho}\left(\frac{e^{-i k_{1} R}}{R}-\frac{e^{-i k_{1} R_{i}}}{R_{i}}+I_{A}\right) \\
I_{A} & =\int_{0}^{\infty} \frac{2 k_{1}{ }^{2} k_{\rho}}{k_{0}{ }^{2} \xi_{1}+k_{1}{ }^{2} \xi_{0}} e^{\xi_{1}(z-h)} J_{0}\left(k_{\rho} \rho\right) d k_{\rho} .
\end{aligned}
$$


Therefore, the three components of the electric field can be obtained in our model:

$$
\begin{aligned}
E_{\rho} & =\frac{\cos \theta I l}{4 \pi\left(\sigma_{1}+i \omega \varepsilon_{1}\right)}\left[k_{1}^{2}\left(\frac{e^{-i k_{1} R}}{R}-\frac{e^{-i k_{1} R_{i}}}{R_{i}}+I_{1 x}\right)\right. \\
& \left.+\frac{\partial^{2}}{\partial \rho^{2}}\left(\frac{e^{-i k_{1} R}}{R}-\frac{e^{-i k_{1} R_{i}}}{R_{i}}+I_{A}\right)\right], \\
E_{\theta} & =-\frac{\sin \theta I l}{4 \pi\left(\sigma_{1}+i \omega \varepsilon_{1}\right)}\left[k_{1}^{2}\left(\frac{e^{-i k_{1} R}}{R}-\frac{e^{-i k_{1} R_{i}}}{R_{i}}+I_{1 x}\right)\right. \\
& \left.+\frac{1}{\rho} \frac{\partial}{\partial \rho}\left(\frac{e^{-i k_{1} R}}{R}-\frac{e^{-i k_{1} R_{i}}}{R_{i}}+I_{A}\right)\right], \\
E_{z} & =\frac{\cos \theta I l}{4 \pi\left(\sigma_{1}+i \omega \varepsilon_{1}\right)} \frac{\partial^{2}}{\partial z \partial \rho}\left(\frac{e^{-i k_{1} R}}{R}+\frac{e^{-i k_{1} R_{i}}}{R_{i}}-\frac{k_{0}^{2}}{k_{1}^{2}} I_{A}\right) .
\end{aligned}
$$

An important problem is how to solve the Sommerfeld integrals in the field expressions. Under the condition of ELF or SLF, the field is in quasi-static situation. In order to facilitate the calculation, the quasi-static approximations can be used. In this case, Wait [17] has shown that $k_{0}{ }^{2} \xi_{1}+k_{1}{ }^{2} \xi_{0} \approx$ $k_{1}^{2} \xi_{0}, \xi_{0}=\sqrt{k_{\rho}^{2}-k_{0}^{2}} \approx k_{\rho}$, and these approximations have been verified available by many scholars, such as Cooray [18]. In addition, a Wait and Spies [19] image theory approximation is utilized here:

$$
\frac{\xi_{1}-k_{\rho}}{\xi_{1}+k_{\rho}} \approx e^{-k_{\rho} d}
$$

where $d=\delta(1-i)$ and $\delta$ is the skin depth given by $\delta=$ $\sqrt{1 / \pi f \mu \sigma_{1}}$.

Thus, by using the quasi-static approximations, it is possible to obtain the approximate expressions of Sommerfeld integrals $I_{1 x}$ and $I_{A}$ as follows:

$$
\begin{aligned}
I_{1 x} & \approx \int_{0}^{\infty} \frac{2 k_{\rho}}{\xi_{1}+k_{\rho}} e^{\xi_{1}(z-h)} J_{0}\left(k_{\rho} \rho\right) d k_{\rho} \\
& \approx \int_{0}^{\infty}\left(1-e^{-k_{\rho} d}\right) e^{\xi_{1}(z-h)} J_{0}\left(k_{\rho} \rho\right) d k_{\rho}=A-B, \\
A & =\int_{0}^{\infty} e^{\xi_{1}(z-h)} J_{0}\left(k_{\rho} \rho\right) d k_{\rho}, \\
B & =\int_{0}^{\infty} e^{-k_{\rho} d} e^{\xi_{1}(z-h)} J_{0}\left(k_{\rho} \rho\right) d k_{\rho}, \\
I_{A} & \approx 2 \int_{0}^{\infty} e^{\xi_{1}(z-h)} J_{0}\left(k_{\rho} \rho\right) d k_{\rho} .
\end{aligned}
$$

Use the approximation that Bannister and Dube [5] have verified as $e^{\xi_{1}(z-h)} \approx e^{i k_{1} m(z-h)} e^{k_{\rho} n(z-h)}$, where the constants $m$ and $n$ get different values in different cases. When $\sqrt{\rho^{2}+(z-h)^{2}} \ll \delta(\delta$ is the skin depth), $m=0$ and $n=1$. When $\sqrt{\rho^{2}+(z-h)^{2}}$ and $\delta$ are of the same order of magnitude, $m=0.4$ and $n=0.96$ for $\sqrt{\rho^{2}+(z-h)^{2}} / \delta<1$ and $m=0.96$ and $n=0.4$ for $1<\sqrt{\rho^{2}+(z-h)^{2}} / \delta<10$. This approximation has a good match presented in the paper written by Gavriloska et al. [20].

Utilizing the Sommerfeld identity [21]

$$
\int_{0}^{\infty} \frac{e^{-\xi(z-h)}}{\xi} J_{0}\left(k_{\rho} \rho\right) k_{\rho} d k_{\rho}=\frac{e^{-i k R}}{R} .
$$

Then

$$
\begin{aligned}
A & \approx e^{i k_{1} m(z-h)} \int_{0}^{\infty} e^{k_{\rho} n(z-h)} J_{0}\left(k_{\rho} \rho\right) d k_{\rho} \\
& =\frac{e^{-i k_{1} m(-z+h)}}{\sqrt{\rho^{2}+[n(-z+h)]^{2}}}, \\
B & \approx \int_{0}^{\infty} e^{-k_{\rho} d} e^{i k_{1} m(z-h)} e^{k_{\rho} n(z-h)} J_{0}\left(k_{\rho} \rho\right) d k_{\rho} \\
& =\frac{e^{-i k_{1} m(-z+h)}}{\sqrt{\rho^{2}+[n(-z+h)+d]^{2}}}, \\
I_{A} & \approx 2 e^{i k_{1} m(z-h) \int_{0}^{\infty} e^{k_{\rho} n(z-h)}} J_{0}\left(k_{\rho} \rho\right) d k_{\rho} \\
& =\frac{2 e^{-i k_{1} m(-z+h)}}{\sqrt{\rho^{2}+[n(-z+h)]^{2}}} .
\end{aligned}
$$

Thus, the three components of the electric field can be defined by more easily calculated expressions with our efforts, which eliminate the difficulties in computing the Sommerfeld integrals. To obtain the electric field, which should be done first, is to calculate the current distribution along the antenna. Take the field point coordinates and the source point coordinates which are, respectively, $(x, y, z)$ and $\left(x^{\prime}, y^{\prime}, z^{\prime}\right)$. The horizontal antenna is oriented along $x$-axis, and the coordinates of its center point are $\left(0,0, z^{\prime}\right)$. Considering that the current is distributed on the antenna surface only, the $x$ component of the electric field generated by current is $\mathbf{E}_{x}$ while the $x$ component of the electric field generated by other sources is $\mathbf{E}_{x}^{i}$. Because the antenna surface is considered to be an ideal conductor, according to the boundary conditions of the ideal conductor, the following equation should be satisfied:

$$
\mathbf{E}_{x}(\mathbf{s})+\mathbf{E}_{x}^{i}(\mathbf{s})=0 .
$$

Since the antenna radius $a$ and the wavelength $\lambda$ meet the condition $a \ll \lambda$, the assumption that current is uniformly distributed along the surface is defined. Then the effect of the current along the antenna surface is equivalent to a line current along $x$-axis.

The MOM [22] is used to solve the current distribution, and in this case the MOM formula of the horizontal antenna 
is as shown in (28). In order to get the numerical result, pulse base and point matching method are chosen in this section:

$$
\begin{aligned}
\mathbf{E}_{x}^{i} & =-\frac{I l}{4 \pi\left(\sigma_{1}+i \omega \varepsilon_{1}\right)} \int_{l} I\left(x^{\prime}\right) \\
\cdot & {\left[k_{1}^{2}\left(\frac{e^{-i k_{1} R}}{R}-\frac{e^{-i k_{1} R_{i}}}{R_{i}}+I_{1 x}\right)\right.} \\
& \left.+\frac{\partial^{2}}{\partial x^{2}}\left(\frac{e^{-i k_{1} R}}{R}-\frac{e^{-i k_{1} R_{i}}}{R_{i}}+I_{A}\right)\right] d x^{\prime},
\end{aligned}
$$

where $R=\sqrt{a^{2}+\left(x-x^{\prime}\right)^{2}}$ and $R_{i}=\sqrt{a^{2}+\left(x+x^{\prime}\right)^{2}}$.

Already knowing the current distribution, the three components of the electric field are easily computed by utilizing formulas (22). The voltage is a parameter that can be directly measured, so we should obtain the voltage received on the ground. Formula (29) shows the expression of the voltage received on the ground by making use of $E_{\rho}$ :

$$
V_{r}=\sum_{i}^{N} \frac{E_{\rho}\left(\rho_{i}\right)+E_{\rho}\left(\rho_{i+1}\right)}{2}\left(\rho_{i+1}-\rho_{i}\right),
$$

where $V_{r}$ is the voltage received on the ground and $\rho_{i}$ is the distance of the $i$ th point.

\section{Numerical Results and Discussion}

3.1. Field of the Antenna. Consider an underground horizontal wire antenna which is located $500 \mathrm{~m}$ down from the ground surface as the signal generator. The ground is seen as homogenous medium, and the characters are $\sigma=0.075 \mathrm{~S} / \mathrm{m}$, $\varepsilon=20 \varepsilon_{0}=1.7708 \times 10^{-10} \mathrm{~F} / \mathrm{m}$, and $\mu=4 \pi \times 10^{-7} \mathrm{H} / \mathrm{m}$. The length of the antenna is $l=6 \mathrm{~m}$ while the radius is $a=0.05 \mathrm{~m}$. As the feed terminal, there is a $1 \mathrm{~V} \delta$ voltage source excitation added on the center, where the $\delta$ gap is $0.1 \mathrm{~m}$. In the following work, we take the modulus of the complex numbers as the values of the current.

The current distribution of the horizontal antenna is calculated by MOM. Figure 2 shows the current distribution on the wire antenna surface when the frequency is $10 \mathrm{~Hz}$. The current distribution is approximate triangular distribution, which well matches the known current distribution of electrically small antenna.

The electric field on the ground plane is obtained afterwards. The magnitude and the phase of the field are both analyzed to know the field more clearly. Figure 3 shows the curves of the three components of the electric field magnitude along the radial distance while Figure 4 shows the curves of the magnitude at different radial distances with the polar angle in polar coordinates.

The curves in Figure 3(a) represent the variation of the radial component of the electric field along the radial distance in different polar angles. The magnitude of the radial component of the electric field goes through a process that gets smaller to a minimum value from the maximum value first and then gets bigger and afterwards gets smaller gently again along the radial distance in all polar angles. When the polar

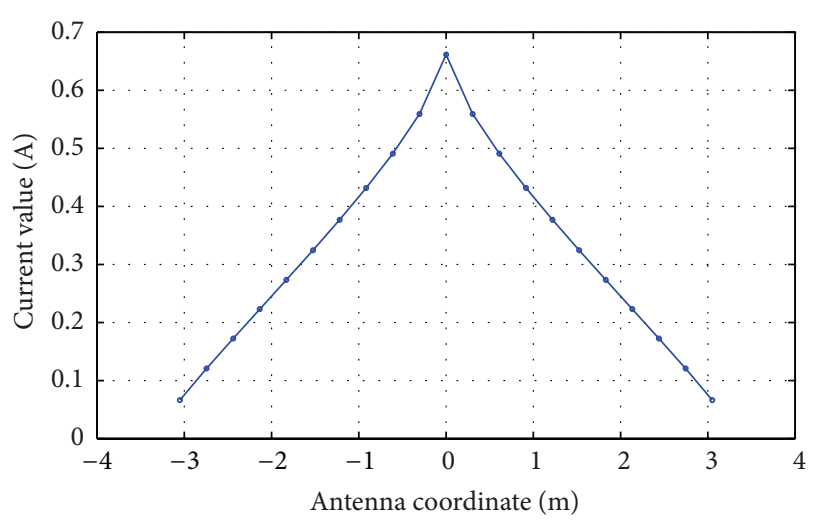

Figure 2: Current distribution on the wire antenna surface.

angle $\theta=0^{\circ}$, the magnitude is larger than the corresponding ones in the same radial distance when the polar angle takes other values. Curves on Figure 3(b) show the variation of the tangential component of the electric field, from which we can see the magnitude gets smaller with the increasing radial distance, and the magnitude reaches maximum when the polar angel $\theta=90^{\circ}$. The axial component of the electric field magnitude becomes larger with the increasing distance first and then becomes smaller after a maximum value, which is shown in Figure 3(c). The trends of the axial component of the electric field with the radial distance at different polar angles are the same, but the value at $\theta=0^{\circ}$ is larger than the others. Compared with the other two components, the axial component of the electric field is several orders of magnitude smaller. The curves of the electric field magnitude with the polar angle are shown in Figure 4; they are symmetrical in the polar coordinates and shaped almost like circles in corresponding half areas.

The phase of the electric field is also studied as shown in Figure 5. The phase of the radial component of the electric field undergoes a mutation at the radial distance between 150 and $200 \mathrm{~m}$, and the other two components get to gradually increase with the increasing radial distance. The phase mutation point is exactly the minimum point of the magnitude, which illustrates the direction of the radial component of the electric field changes in this point. In addition, the trends of the phase variation with the radial distance at different polar angles are similar.

Figure 6 shows the curves about the variation of the total electric field with the radial distance on the ground. The curve generally presents a tendency which the field magnitude decreases with the increasing distance.

The curve of the voltage received on the ground with the radial distance is shown in Figure 7 . Here the original point on the ground is taken as the relative voltage zero point, and the voltage value is gained at the polar angle $\theta=0^{\circ}$. The received voltage value tends to increase first and gets to decrease after a maximum value. The maximum value appears at the point that the direction of the radial component of the electric field begins to change. 


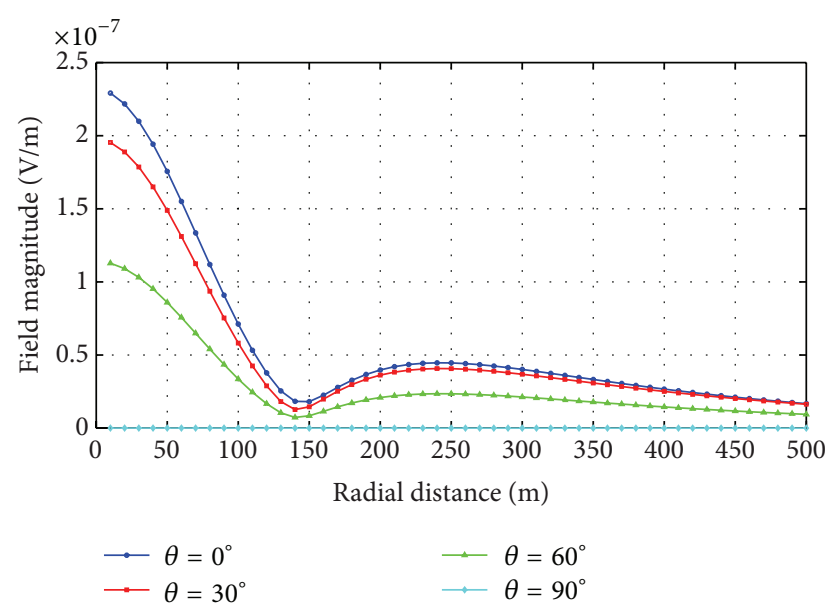

(a)

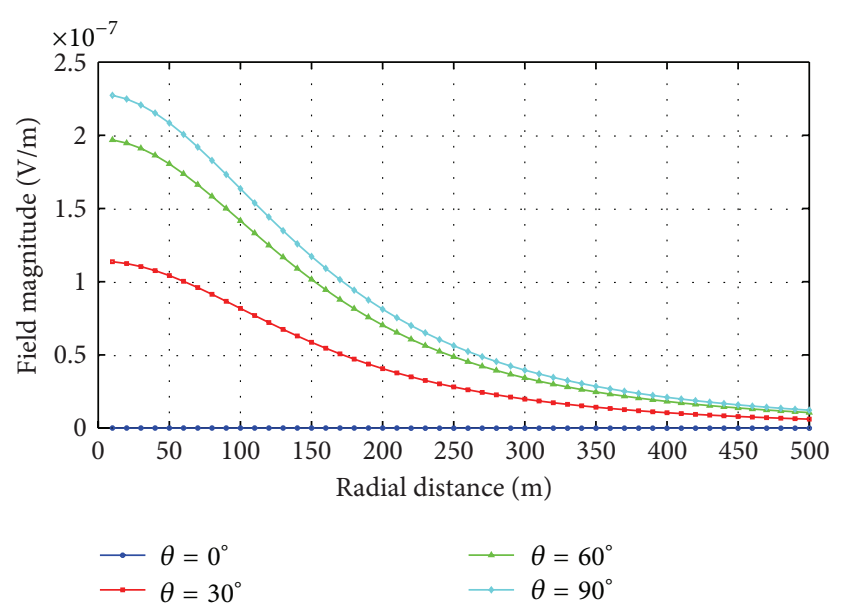

(b)

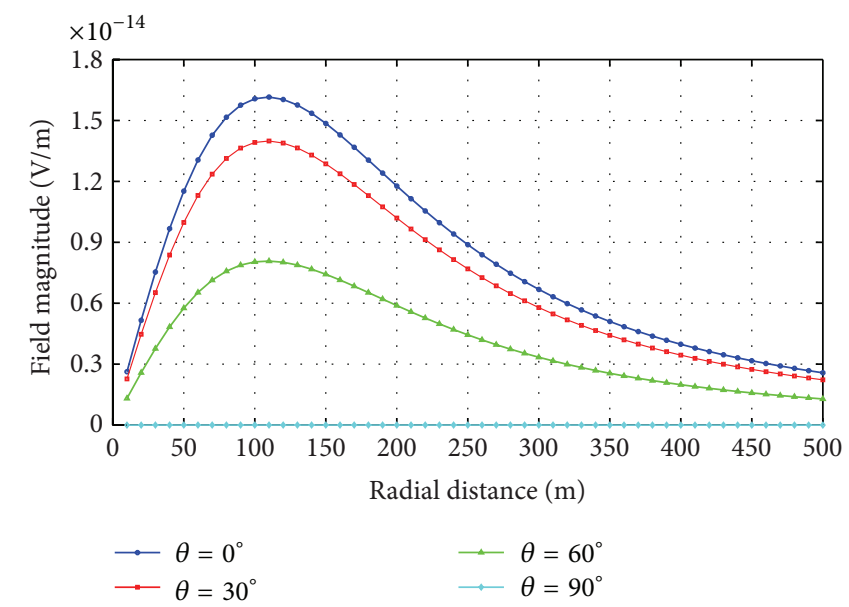

(c)

FIGURE 3: Curves of (a) the radial component of the electric field magnitude, (b) the tangential component of the electric field magnitude, and (c) the axial component of the electric field magnitude with the radial distance.

3.2. Influence of the Buried Depth on Field. The buried depth of the antenna can change the electric field distribution on the ground. In this part, the maximum value of the current on the antenna surface is chosen to study the influence of the buried depth on current distribution. In Figure 8, it is shown that the current value tends to increase with the deepening depth, but the influence is not obvious numerically. The radial component of the electric field is of our most concern, which directly affects the voltage received, so the radial component of the electric field can be a representative to study the influence on electric field. Three points corresponding to the radial distance $R=50 \mathrm{~m}, R=150 \mathrm{~m}$, and $R=250 \mathrm{~m}$ at the polar angle $\theta=0^{\circ}$ are taken as the study objects. As shown in Figure 9, the radial component of the electric field magnitude becomes smaller when the buried depth becomes deeper at different radial distances, which indicates that the deeper the buried depth, the smaller the electric field magnitude in the underground communication system.

3.3. Influence of the Antenna Length on Field. The antenna size is a factor that can influence the electric field. Since the model is a line antenna model, the radius is much smaller than the length, so the study aims at the influence of the antenna length on the electric field. The same study objects as the study on Section 3.2 are used in this case. Figure 10 shows the curve of the maximum current value with the antenna length from $5 \mathrm{~m}$ to $7 \mathrm{~m}$, which demonstrates that the current value has an obvious increase when the antenna length gets longer. Figure 11 represents the curves of the radial component of the electric field magnitude with the antenna length at different radial distance. The data indicates that the field magnitude gets larger at any radial distance with the increasing antenna length. So we can obtain the conclusion that longer antenna will produce better results.

3.4. Influence of the Frequency on Field. In lossy medium, the attenuation of the electric field increases as the frequency gets higher, so the frequency is an important parameter that can influence the electric field on the ground. Similarly, the maximum current value on the antenna surface and the radial component of the electric field magnitude can be the representative to study the influence of the frequency on 


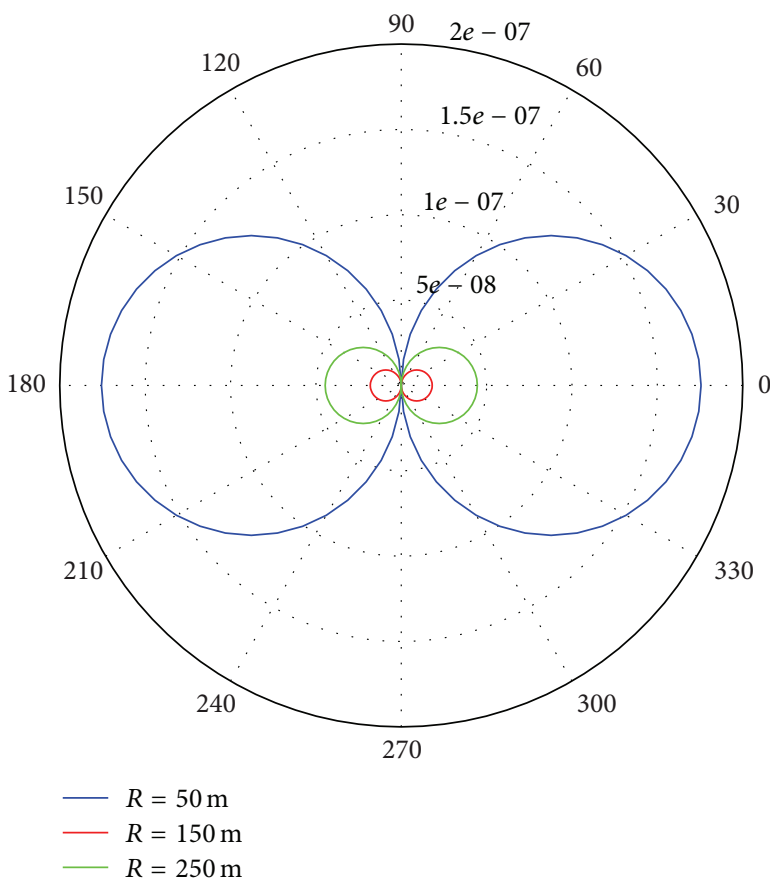

(a)

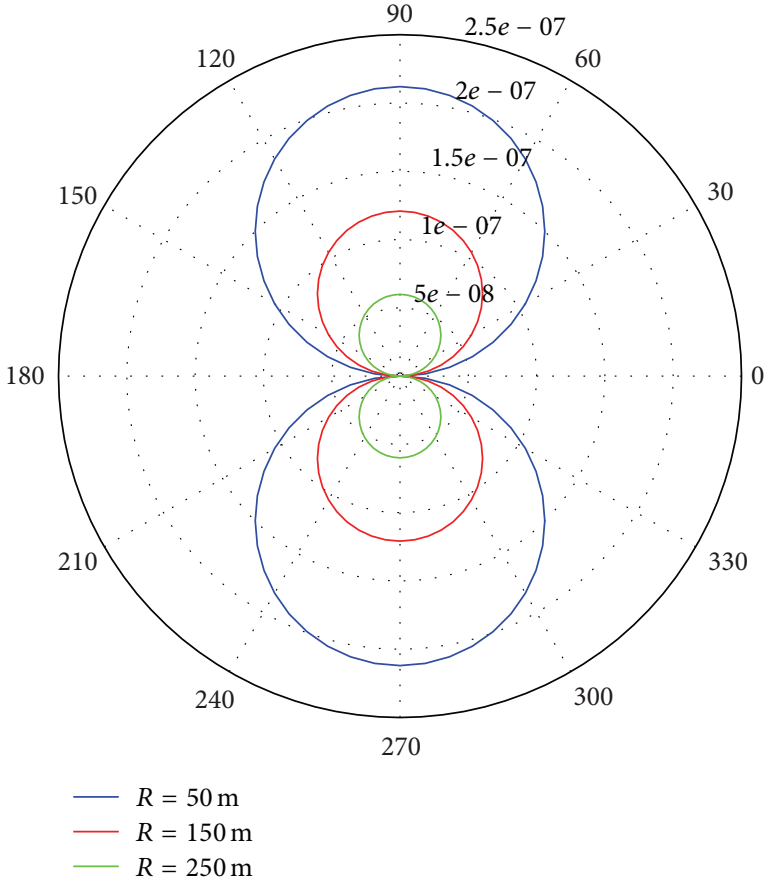

(b)

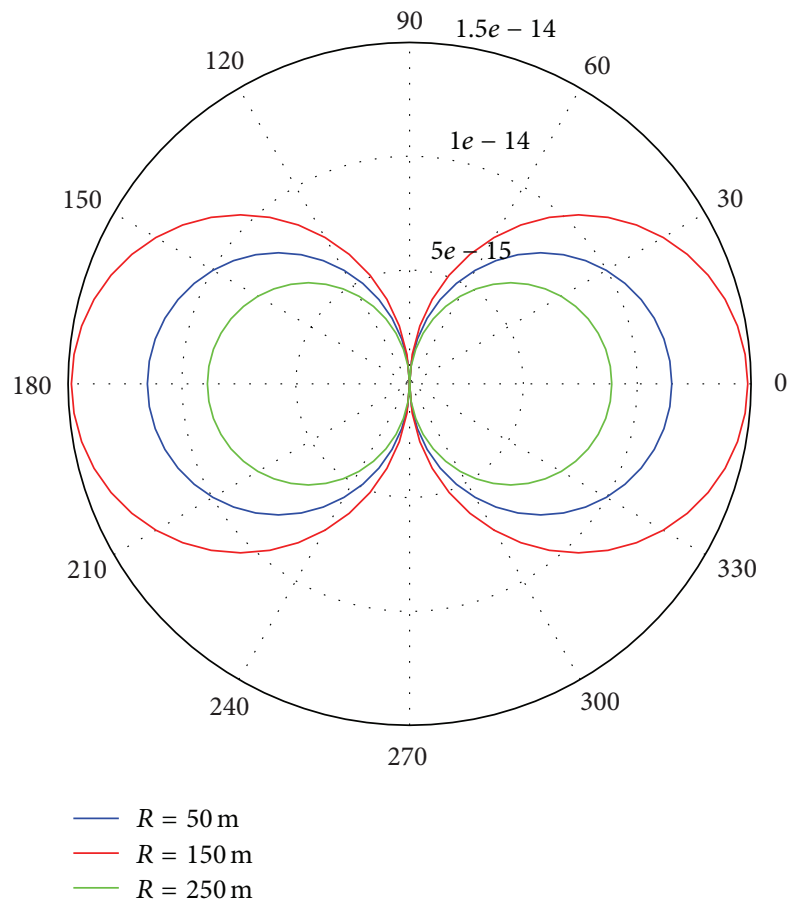

(c)

FIgURE 4: Curves of (a) the radial component of the electric field magnitude, (b) the tangential component of the electric field magnitude, and (c) the axial component of the electric field magnitude with the polar angle.

electric field. In Figure 12, it is shown that the current value tends to decrease with the increasing frequency, but the influence is too tiny to distinguish the size of the value. As shown in Figure 13, the field value at the points whose radial distances $R=50 \mathrm{~m}$ and $R=250 \mathrm{~m}$ decreases when the frequency increases, but the field value at the point $R=150 \mathrm{~m}$ gets a slight increase when the frequency increases. The point $R=150 \mathrm{~m}$ is just in the vicinity of the point where the radial component of the electric field magnitude takes the minimum value and the field phase undergoes a mutation. 


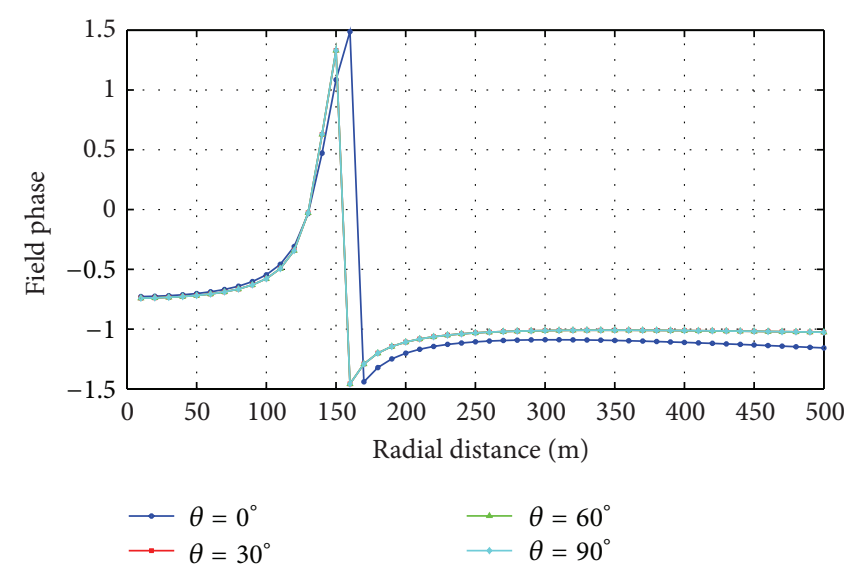

(a)

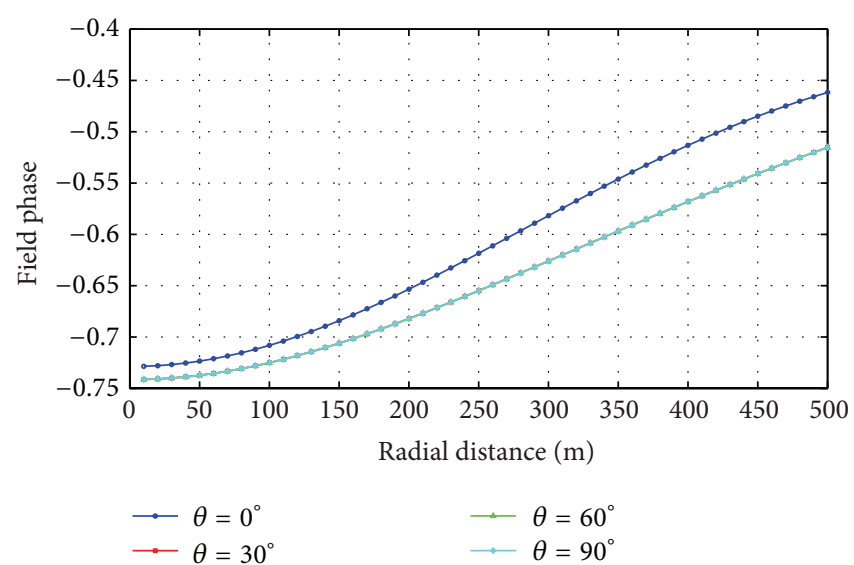

(b)

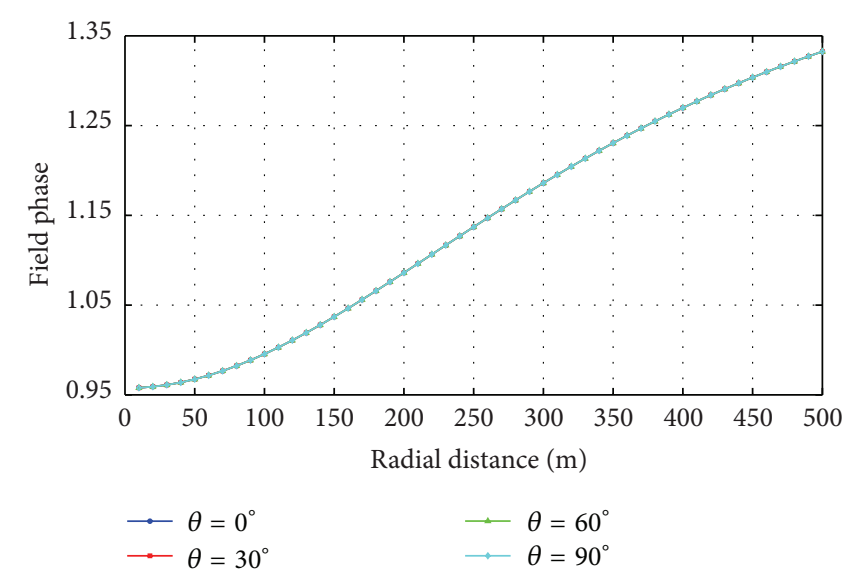

(c)

Figure 5: Curves of (a) the radial component of the electric field phase, (b) the tangential component of the electric field phase, and (c) the axial component of the electric field phase with the radial distance.

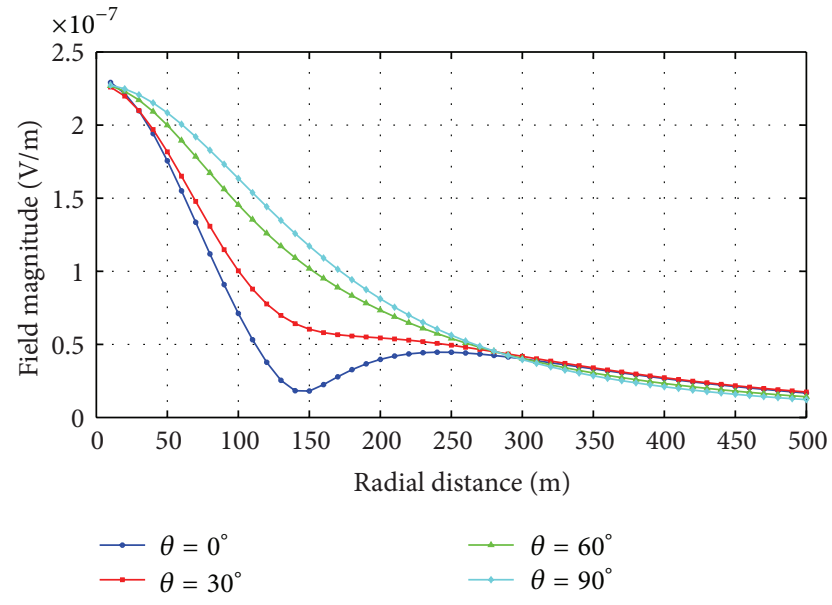

Figure 6: Curves of the total electric field magnitude with the radial distance.

This shows that the radial distance of the mutation point increases with the increasing frequency.

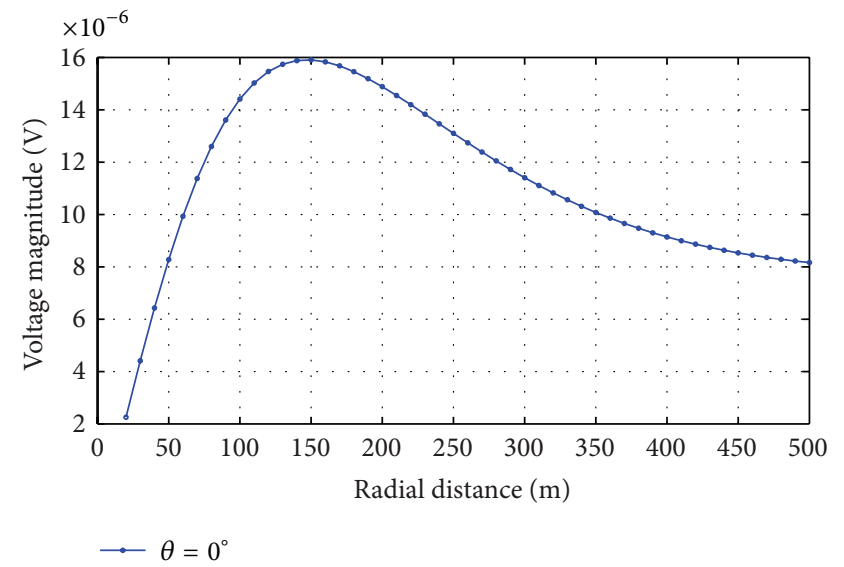

FIGURE 7: Curve of the voltage received on the ground with the radial distance.

3.5. Influence of the Stratum Parameters on Field. The characteristic of the formation affects the field as well, two of which are the stratum conductivity and the relative permittivity. 


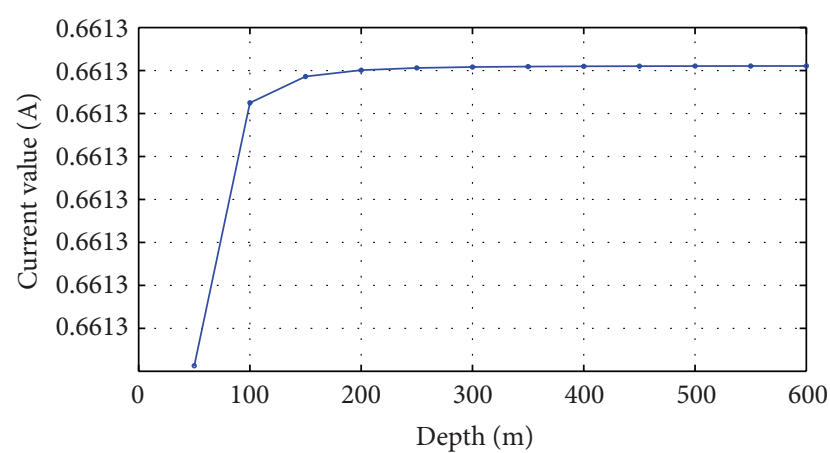

FIGURE 8: Curve of the maximum current value with the buried depth.

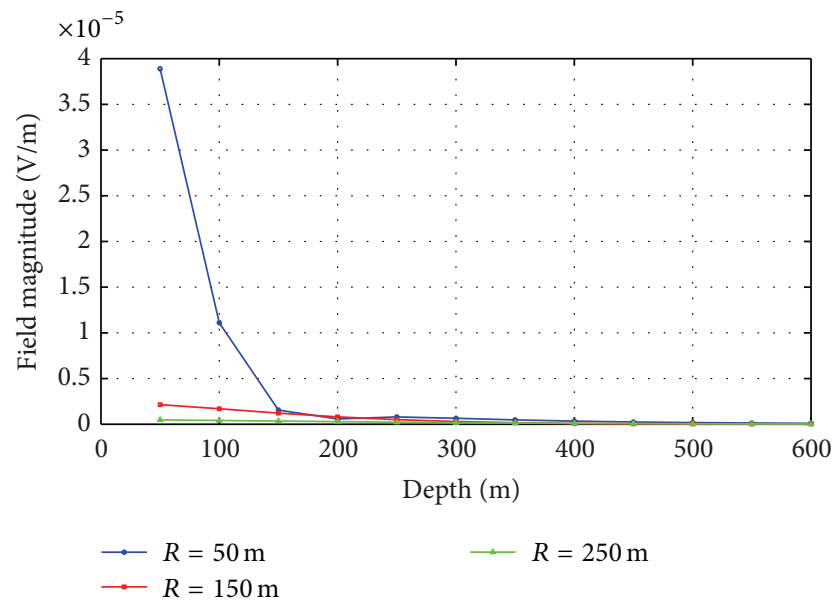

FIGURE 9: Curves of the radial component of the electric field magnitude with the buried depth.

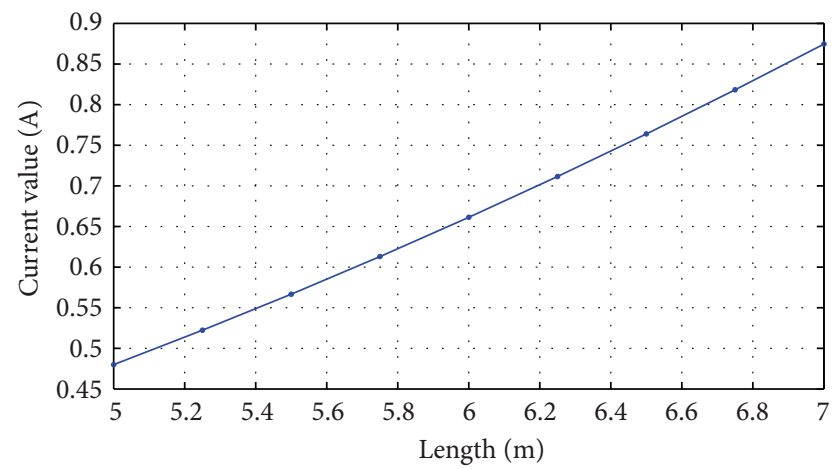

FIgURE 10: Curve of the maximum current value with the antenna length.

Similar with the previous study, we choose the maximum current value on antenna surface as the object to study the influence on current and use the radial component of the electric field magnitude corresponding to the points $R=50 \mathrm{~m}$, $R=150 \mathrm{~m}$, and $R=250 \mathrm{~m}$ at the polar angle $\theta=0^{\circ}$ to study the influence on electric field. Figure 14 shows the curve of current value varying with the conductivity, which indicates that the current value has a positive correlation with the

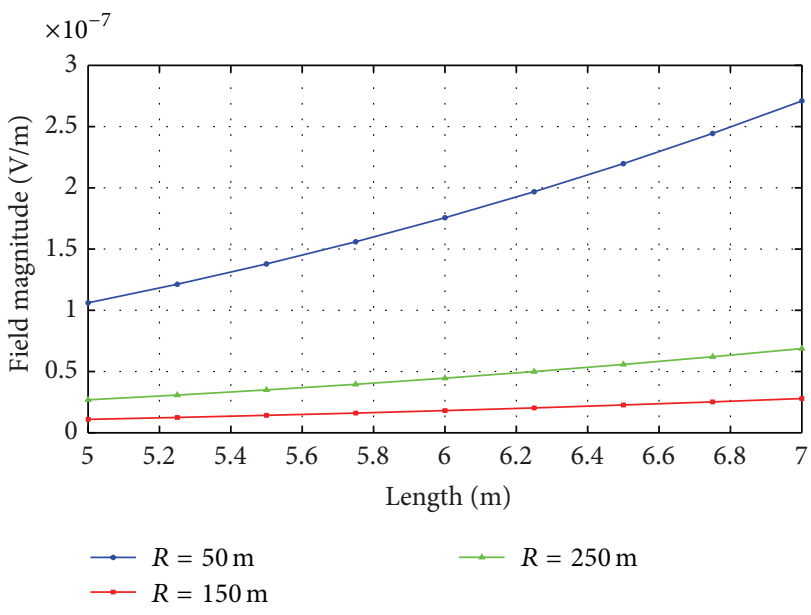

Figure 11: Curves of the radial component of the electric field magnitude with the antenna length.

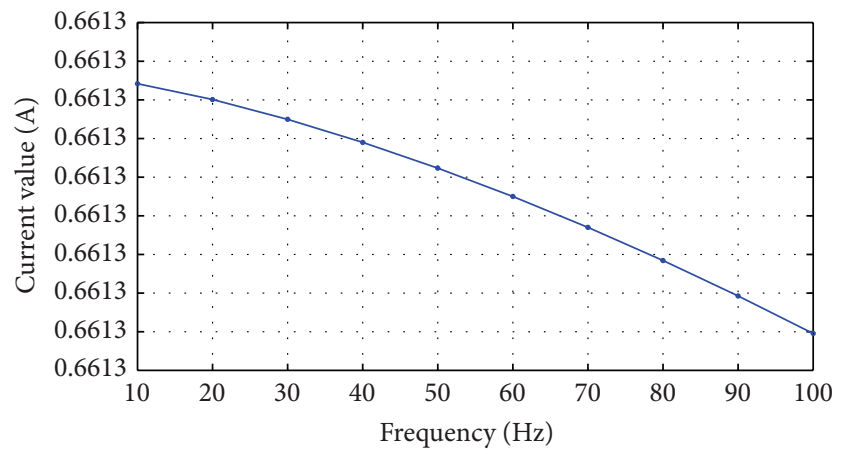

FIGURE 12: Curve of the maximum current value with the frequency.

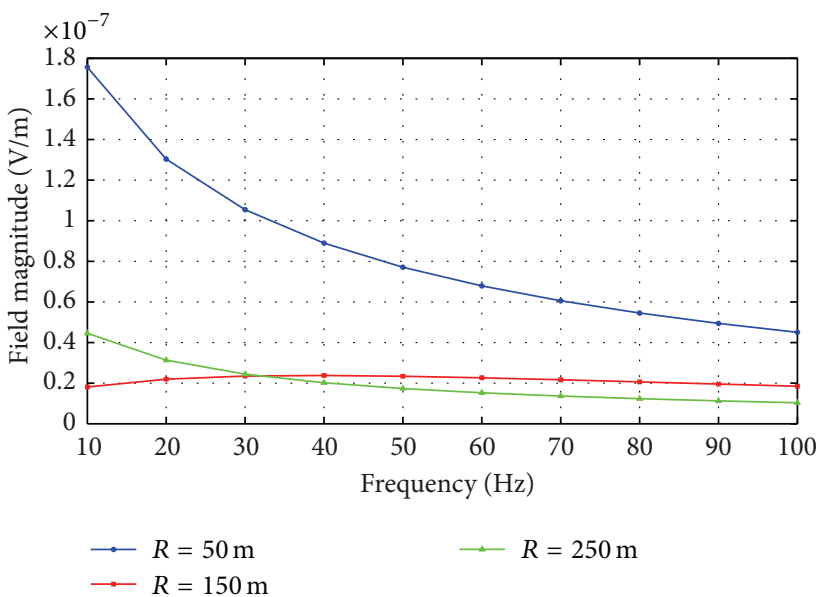

FIGURE 13: Curves of the radial component of the electric field magnitude with the frequency.

conductivity. Curves in Figure 15 demonstrate the variation of the radial component of the electric field magnitude with the conductivity from 0.05 to $0.1 \mathrm{~S} / \mathrm{m}$ at the three points selected. With the increasing conductivity, the field magnitudes at the three points all get smaller. There are obvious variations at the 


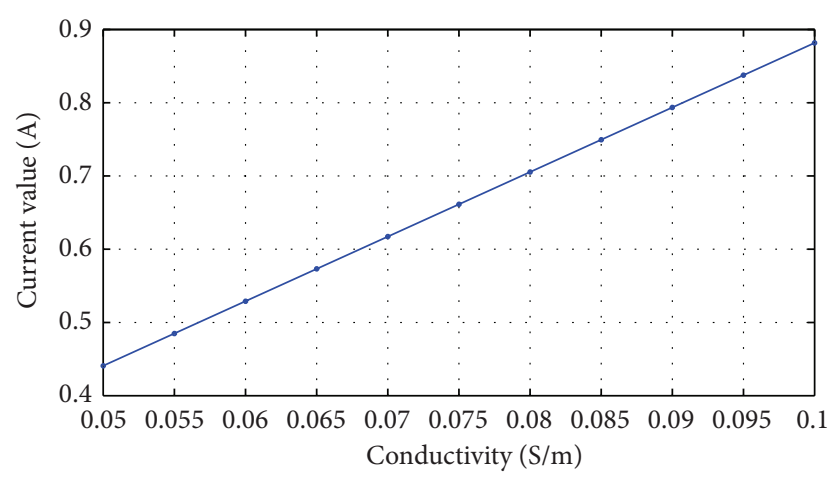

FIGURE 14: Curve of the maximum current value with the conductivity.

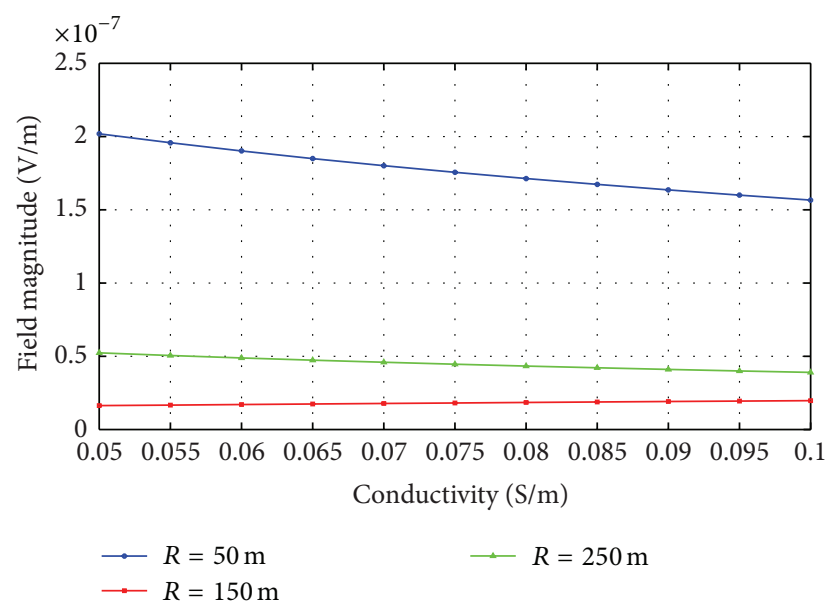

Figure 15: Curves of the radial component of the electric field magnitude with the conductivity.

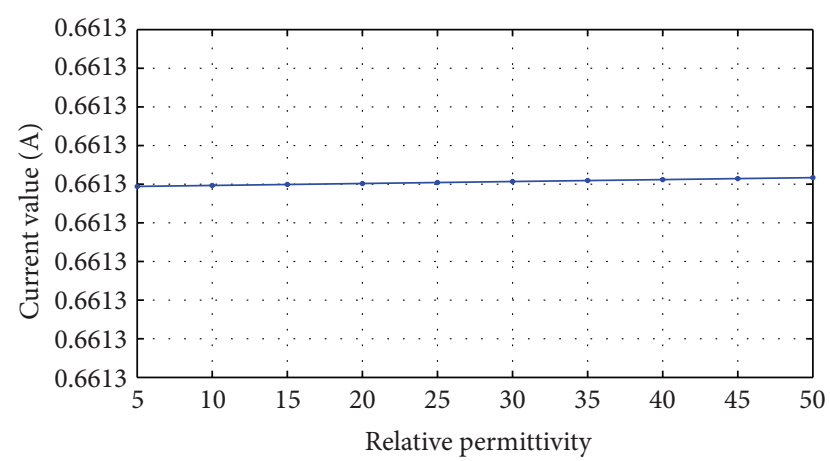

FIGURE 16: Curve of the maximum current value with the relative permittivity.

points $R=50 \mathrm{~m}$ and $R=250 \mathrm{~m}$ and unconspicuous variation at the point $R=150 \mathrm{~m}$.

The effect of the relative permittivity on current and electric field is studied later. Curve in Figure 16 represents the variation of the current value on antenna surface with the relative permittivity. The current value increases when the relative permittivity gets larger, but the influence is so

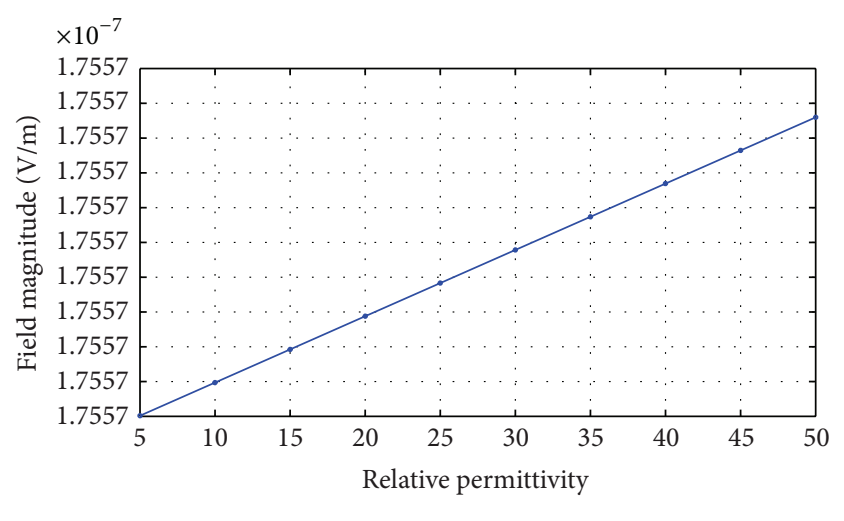

$\rightarrow R=50 \mathrm{~m}$

FIgURE 17: Curve of the radial component of the electric field magnitude with the relative permittivity.

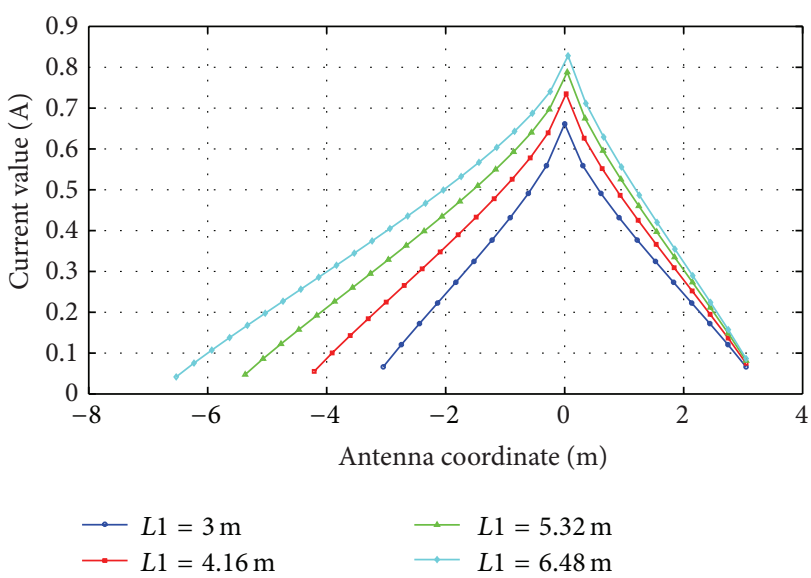

FIGURE 18: Current distribution on the asymmetric antenna surface.

tiny that the change is not obvious. Figure 17 represents the dependence of the radial component of the electric field with the relative permittivity corresponding to the point $R=50 \mathrm{~m}$ at the angle $\theta=0^{\circ}$. The value of the radial component of the electric field is positively correlated with the relative permittivity, and the variation is too tiny to recognize the difference of the value as well.

3.6. Study on the Asymmetric Horizontal Antenna. In the practical application, such as trenchless technology, the antenna used in the underground communication system may be asymmetric. So the asymmetric horizontal antenna is an important subject needed to study. Take one antenna arm length as $3 \mathrm{~m}, 4.16 \mathrm{~m}, 5.32 \mathrm{~m}$, and $6.48 \mathrm{~m}$, respectively, and the other antenna arm length as fixed $3 \mathrm{~m}$, and the current distribution on the antenna surface is calculated. The curves in Figure 18 describe the variation of the current value on the antenna surface with different antennas. The current distribution is nearly triangular distribution no matter whether the antenna is symmetrical, and the distribution is asymmetric on asymmetric antenna. The current value gets bigger when 

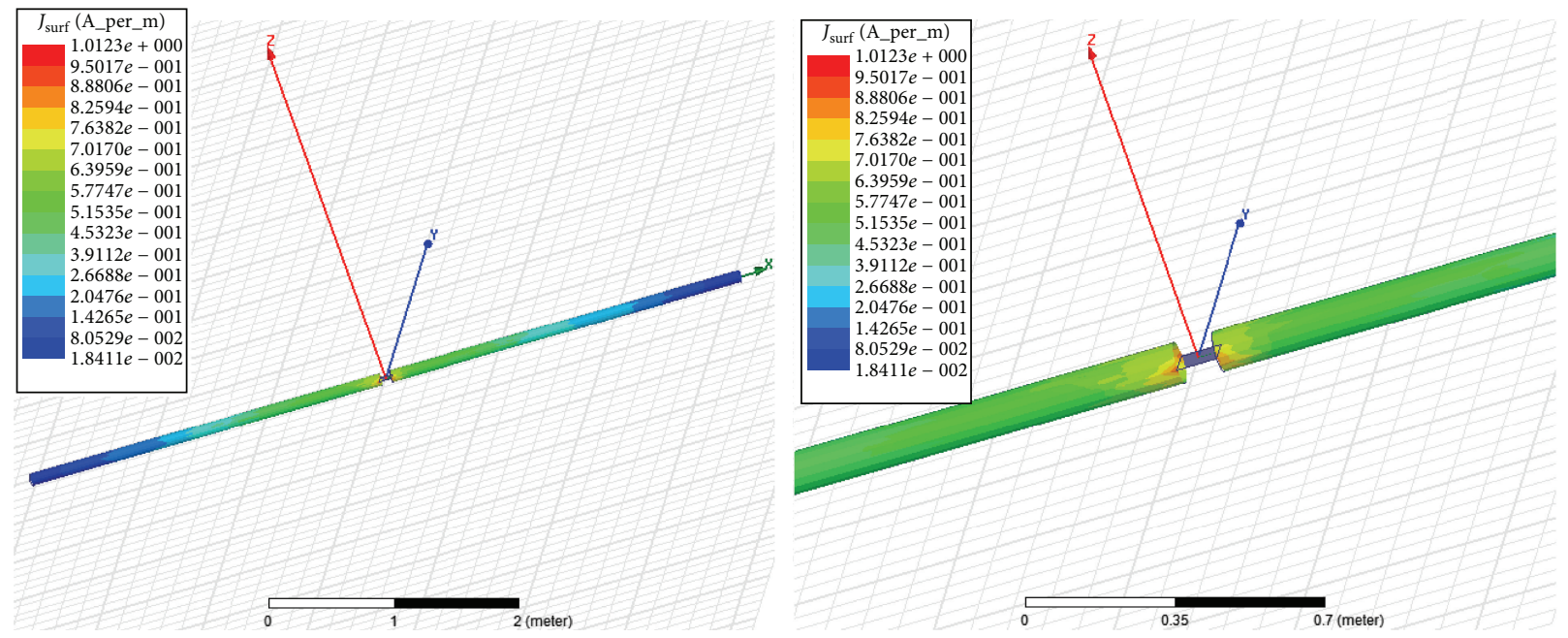

FIGURE 19: Current distribution on the antenna surface by HFSS.

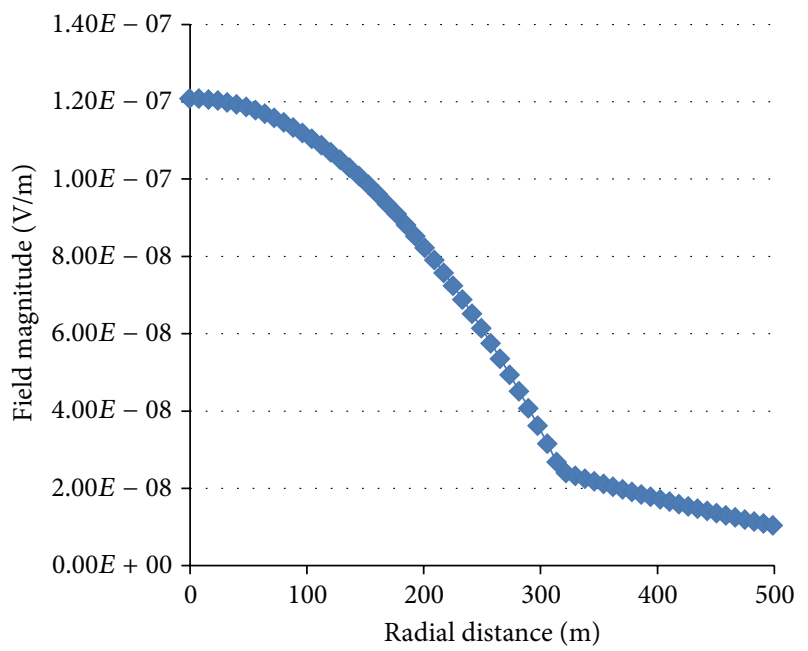

FIGURE 20: Curve of the total electric field with the radial distance by HFSS.

the antenna length gets longer, and the change is relatively obvious.

3.7. Verification. In order to verify the numerical results, a simulation work has been done by using the commercial software HFSS. The model simulated is the same as the previous study with $10 \mathrm{~Hz}$ frequency. The ground characters are $\sigma=0.075 \mathrm{~S} / \mathrm{m}, \varepsilon=20 \varepsilon_{0}=1.7708 \times 10^{-10} \mathrm{~F} / \mathrm{m}$, and $\mu=4 \pi \times 10^{-7} \mathrm{H} / \mathrm{m}$. The current distribution on antenna surface is presented in Figure 19, which matches well with the previous result in Figure 2. Curve on Figure 20 shows the variation of the total field along the radial distance. The electric field value in this case is a little smaller than the result in Figure 6. This consequence may be due to the fact that more factors are concerned in HFSS and the model in this work is too large to calculate. Overall, this HFSS case is largely consistent with the previous results as verification.

\section{Conclusion}

In this work, a more accurate model of horizontal electrically small antenna is established to solve the problem of through the earth communication. The expressions of the electric field generated by an underground horizontal antenna are derived in forms which can be easily solved in this mathematical model. Based on this work, numerical method is used to obtain the current distribution and the electric field on the ground in the condition of $10 \mathrm{~Hz}$. In these numerical results, the variations of three components of the electric field with the radial distance at different polar angles are investigated, respectively. The radial and axial components of the electric field get the maximum value at the polar angle $\theta=0^{\circ}$, and the tangential component of the electric field gets the maximum value at the polar angle $\theta=90^{\circ}$. With the increasing radial distance, the radial component of the electric field at the polar angle $\theta=0^{\circ}$ decreases first and achieves the minimum value at the phase mutation point $R=150 \mathrm{~m}$ and then makes a change of getting larger and afterwards smaller again. The tangential component of the electric field at the polar angle $\theta=90^{\circ}$ diminishes with the radial distance, and the axial component of the electric field at the polar angle $\theta=0^{\circ}$ appears as a trend that increases first and decreases afterwards with the increasing radial distance. In addition, the voltage received on the ground is obtained. At the polar angle $\theta=0^{\circ}$, the maximum value of the voltage is approximately at the radial distance $R=150 \mathrm{~m}$.

In order to have a relatively overall understanding of the antenna, the influence of the buried depth, antenna length, frequency, and stratum parameters on field is studied. The consequence shows that the buried depth, antenna length, conductivity, and relative permittivity are positively related to the current value while the frequency is negatively related to the current. The influence of the antenna length and conductivity on the current value is obvious. The study of these influence factors on the electric field shows that the antenna length and relative permittivity have a positive correlation with the radial component of the electric field magnitude, and 
the buried depth, frequency, and conductivity have a negative correlation with that. The influence of the relative permittivity is very slight. Additionally, the current distribution on the asymmetric horizontal antenna surface is studied.

\section{Conflict of Interests}

The authors declare that there is no conflict of interests regarding the publication of this paper.

\section{Acknowledgment}

This work is supported by the China Geological Survey Program (no. KZ11Z245).

\section{References}

[1] A. Sommerfeld, "Über die Ausbreitung der Wellen in der drahtlosen Telegraphie," Annalen der Physik, vol. 333, no. 4, pp. 665-736, 1909.

[2] A. Sommerfeld, "Über die Ausbreitung der Wellen in der drahtlosen Telegraphie," Annalen der Physik, vol. 386, no. 25, pp. 1135-1153, 1926.

[3] R. K. Moore and W. E. Blair, "Dipole radiation in a conducting half-space," Journal of Research of the National Bureau of Standards-D. Radio Propagation, vol. 65, no. 6, pp. 547-563, 1961.

[4] P. R. Bannister, "The image theory quasi-static fields of antennas above the earth's surface," DTIC Document, 1969.

[5] P. R. Bannister and R. L. Dube, "Simple expressions for horizontal electric dipole quasi-static range subsurface-to-subsurface and subsurface-to-air propagation," Radio Science, vol. 13, no. 3, pp. 501-507, 1978.

[6] P. R. Bannister, "Summary of image theory expressions for the quasi-static fields of antennas at or above the earth's surface," Proceedings of the IEEE, vol. 67, no. 7, pp. 1001-1008, 1979.

[7] D. C. Chang and J. R. Wait, "Extremely low frequency (ELF) propagation along a horizontal wire located above or buried in the earth," IEEE Transactions on Communications, vol. 22, no. 4, pp. 421-427, 1974.

[8] R. W. P. King, "Electromagnetic field of a vertical dipole over an imperfectly conducting half-space," Radio Science, vol. 25, no. 2, pp. 149-160, 1990.

[9] R. W. P. King, C. W. Harrison Jr., and V. A. Houdzoumis, "Electromagnetic field in the sea due to an omnidirectional VLF antenna," Radio Science, vol. 32, no. 1, pp. 103-112, 1997.

[10] C. T. Tai and R. E. Collin, "Radiation of a Hertzian dipole immersed in a dissipative medium," IEEE Transactions on Antennas and Propagation, vol. 48, no. 10, pp. 1501-1506, 2000.

[11] R. Thottappillil, M. A. Uman, and N. Theethayi, "Electric and magnetic fields from a semi-infinite antenna above a conducting plane," Journal of Electrostatics, vol. 61, no. 3-4, pp. 209-221, 2004.

[12] P. K. Vong, D. Rodger, and A. Marshall, "Modeling an electromagnetic telemetry system for signal transmission in oil fields," IEEE Transactions on Magnetics, vol. 41, no. 5, pp. 2008-2011, 2005.

[13] X.-B. Xu and Y. F. Huang, "An efficient analysis of vertical dipole antennas above a lossy half-space," Progress in Electromagnetics Research, vol. 74, pp. 353-377, 2007.
[14] A. Khalatpour, R. Sarraf Shirazi, and G. Moradi, "Analysis of vertical wire antennas above lossy half-space using matrix pencil method," AEU: International Journal of Electronics and Communications, vol. 64, no. 8, pp. 784-789, 2010.

[15] M. Parise, "Second-order formulation for the quasi-static field from a vertical electric dipole on a lossy half-space," Progress in Electromagnetics Research, vol. 136, pp. 509-521, 2013.

[16] P. DeGauque and R. Grudzinski, "Propagation of electromagnetic waves along a drillstring of finite conductivity," SPE Drilling Engineering, vol. 2, no. 2, pp. 127-134, 1987.

[17] J. R. Wait, "The electromagnetic fields of a horizontal dipole in the presence of a conducting half-space," Canadian Journal of Physics, vol. 39, no. 7, pp. 1017-1028, 1961.

[18] V. Cooray, "Horizontal electric field above- and underground produced by lightning flashes," IEEE Transactions on Electromagnetic Compatibility, vol. 52, no. 4, pp. 936-943, 2010.

[19] J. R. Wait and K. P. Spies, "On the image representation of the quasi-static fields of a line current source above the ground," Canadian Journal of Physics, vol. 47, no. 23, pp. 2731-2733, 1969.

[20] O. Gavriloska, M. Mircev, V. Arnautovski, and L. Grcev, "Approximate image formulation for the fields of a horizontal Hertzian dipole in homogenous soil," in Proceedings of the International PhD-Seminar, Numerical Field Computation and Optimization in Electrical Engineering, pp. 89-94, Ohrid, Macedonia, September 2005.

[21] J. A. Stratton, Electromagnetic Theory, John Wiley \& Sons, 2007.

[22] R. F. Harrington and J. L. Harrington, Field Computation by Moment Methods, Oxford University Press, 1996. 

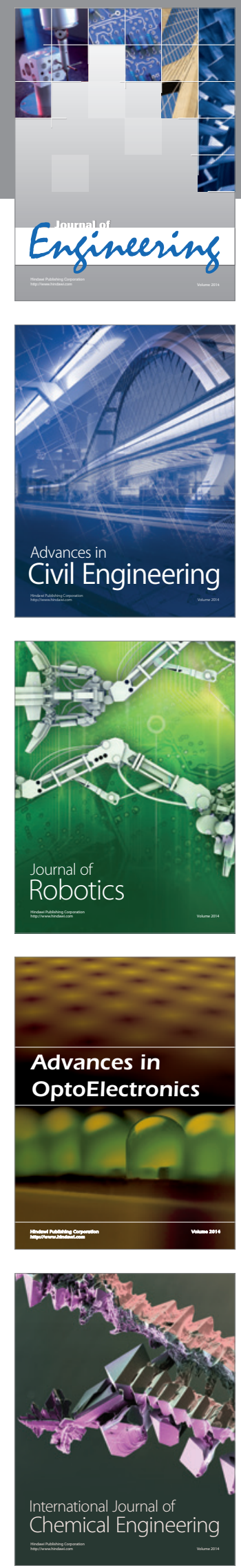

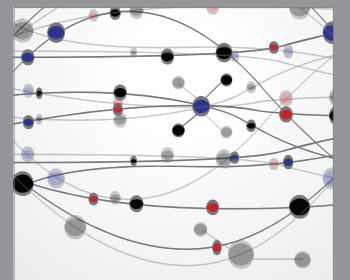

The Scientific World Journal
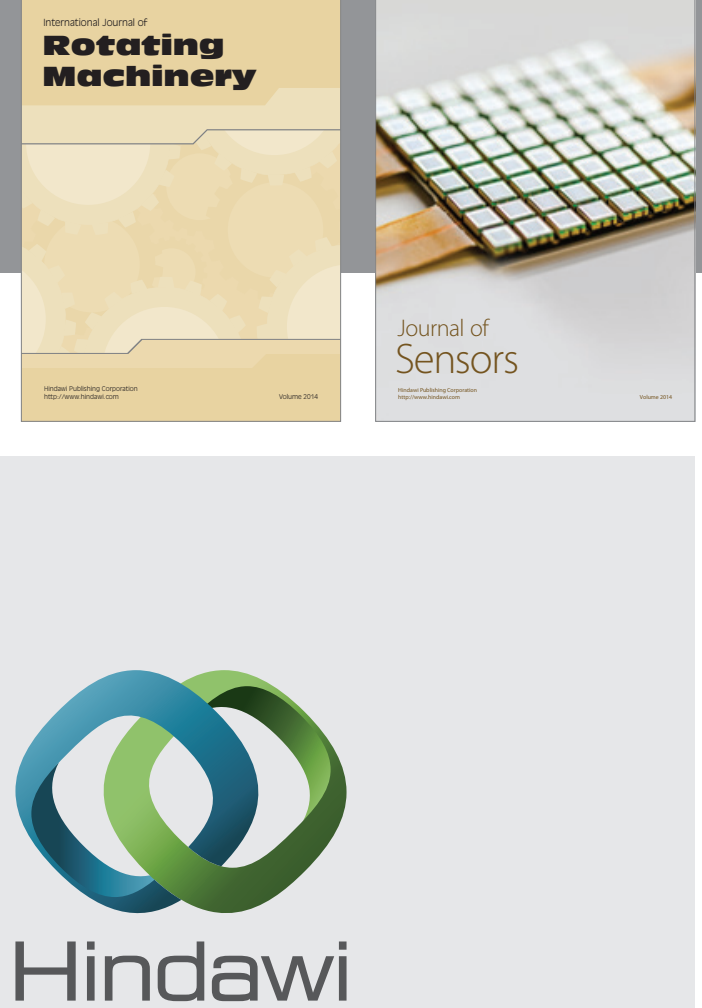

Submit your manuscripts at http://www.hindawi.com
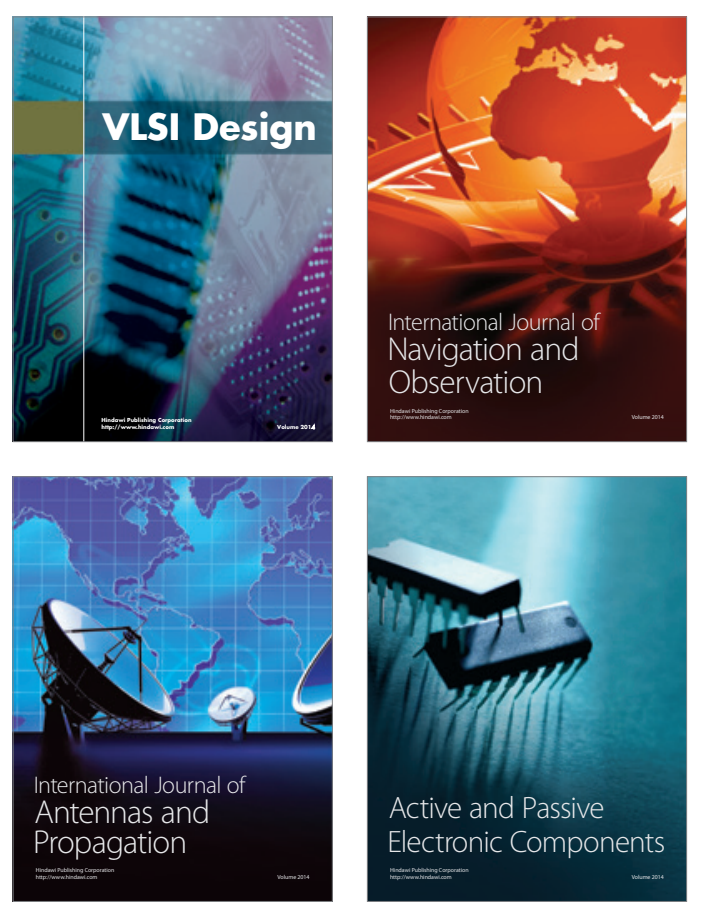
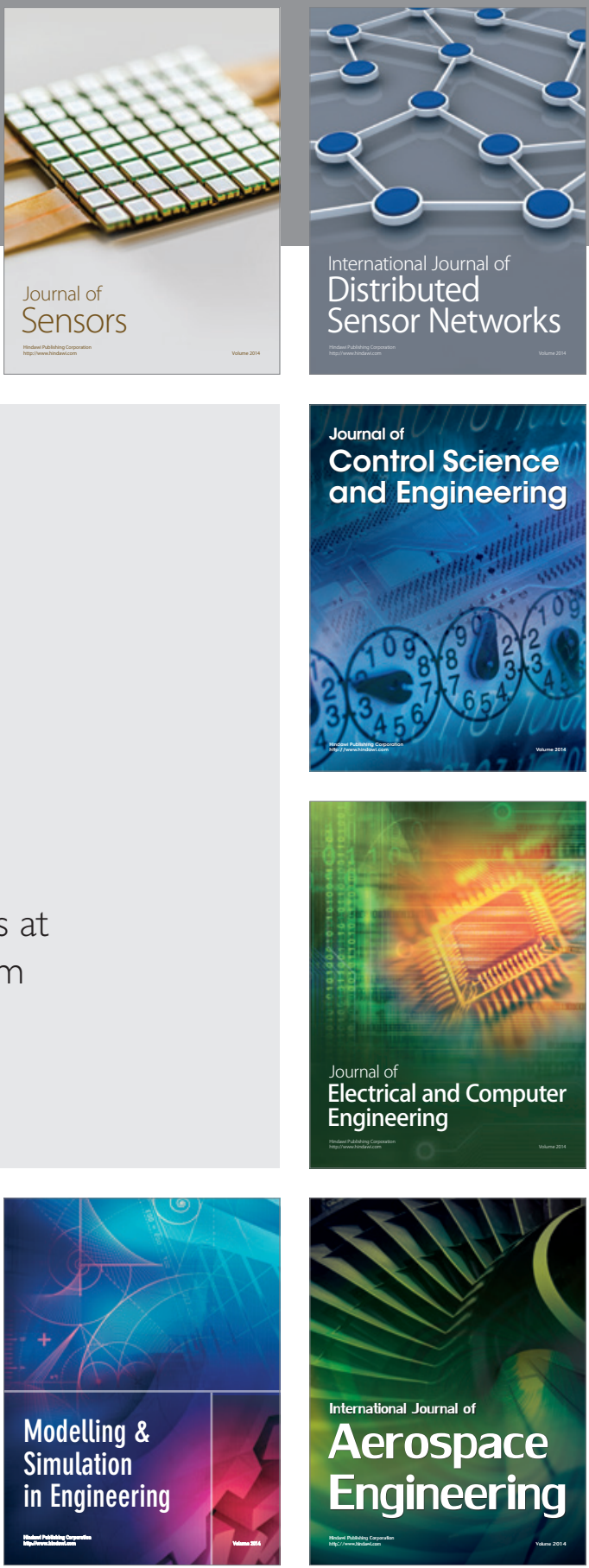

Journal of

Control Science

and Engineering
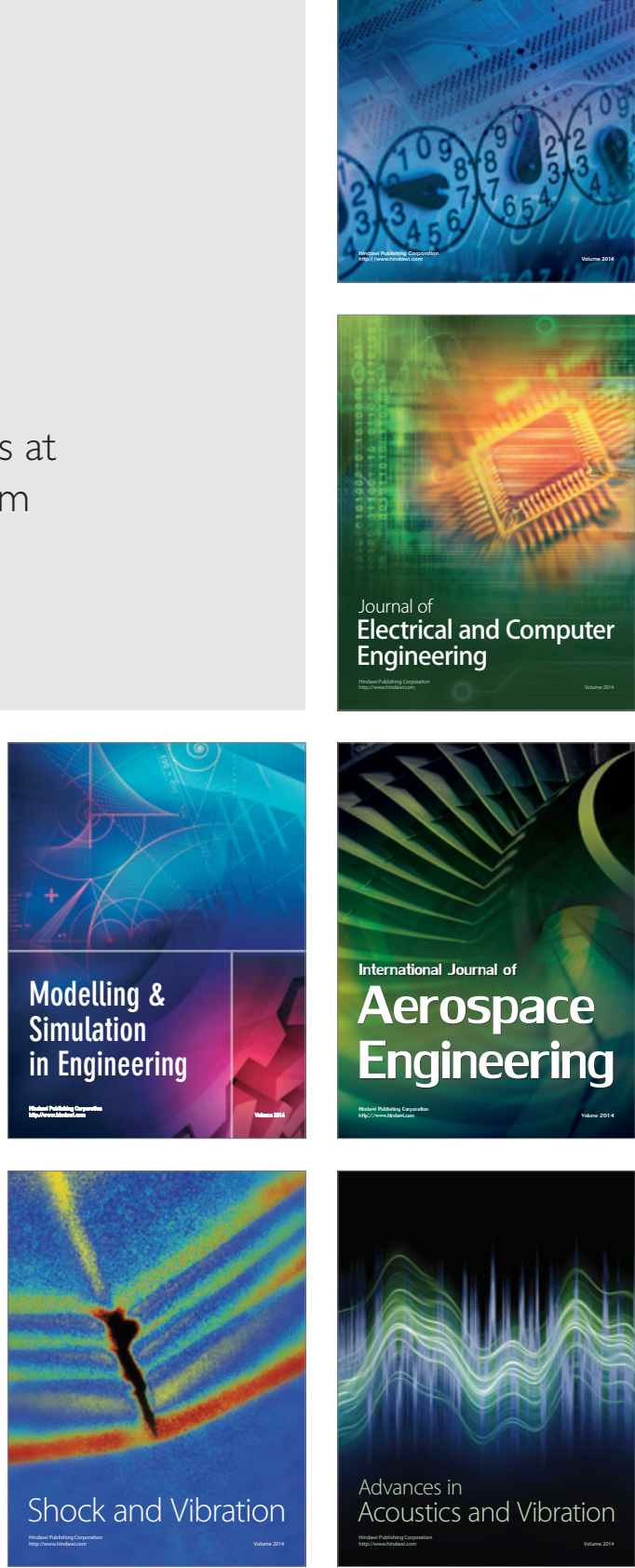